Rev. High Pressure Sci. Technol., Vol. 7 (1998) 1043 1045

\title{
High Pressure Phase Transition of $\mathrm{CrSb}_{2}$
}

\author{
H. Takizawa, K. Uheda, T. Endo, and M. Shimada ${ }^{\dagger}$ \\ Department of Molecular Chemistry and Engineering, Tohoku University, Sendai 980-77, Japan \\ ${ }^{\dagger}$ Institute for Advanced Materials Processing, Tohoku University, Sendai 980-77, Japan
}

\begin{abstract}
Anew high-pressure phase of $\mathrm{CrSb}_{2}$ has been synthesized from the marcasite-type ambient pressure phase by high-pressure/ temperature treatment above $5.5 \mathrm{GPa}$. The crystal structure of the high-pressure phase is body-centered tetragonal, which is assigned as $\mathrm{CuAl}_{2}$-type structure. The bond nature in the high-pressure phase is metallic, in contrast to the mixed ionic and covalent character in the marcasite-type phase. The phase transition, marcasite-type to $\mathrm{CuAl}_{2}-\mathrm{type}$, accompanies $11 \%$ volume decrease and a change of d-electron character from localized to itinerant.

[high-pressure synthesis, phase transition, marcasite-type, $\mathrm{CuAl}_{2}$-type, pnictides]
\end{abstract}

\section{Introduction}

Transition metal dichalcogenides and dipnictides $\left(\mathrm{TX}_{2}\right)$ with the pyrite, marcasite, and arsenopyrite-type structures have been widely discussed on their structural relationship and chemical bonding schemes. The common features of these closely related compounds are the formation of X-X dimer (dumb-bells) and the mixed ionic and covalent character of the chemical bonds $[1,2]$. The $\mathrm{X}-\mathrm{X}$ bond distances in these compounds are nearly equal to sum of the single bond tetrahedral covalent radii. Transition metal atoms are in $\mathrm{T}^{2+}$ (dichalcogenides) or $\mathrm{T}^{4+}$ (dipnictides) valency states and octahedrally coordinated by six chalcogen or pnicogen atoms.

Two types of pressure-induced phase transitions, marcasite (or arsenopyrite) to pyrite and pyrite to marcasite structures, were reported in this family. The former was found in $\mathrm{FeSe}_{2}$, $\mathrm{FeTe}_{2}, \mathrm{CoTe}_{2}, \mathrm{CuSe}_{2}, \mathrm{NiP}_{2}, \mathrm{NiAs}_{2}$ and described to be due to the effective packing of $\mathrm{TX}_{6}$ octahedra $[3,4]$. The latter case was observed in high-pressure X-ray diffraction study of $\mathrm{MnS}_{2}$ and $\mathrm{MnTe}_{2}$ [5-7]. The 15-18\% volume decrease at the transition suggest a high-spin to low-spin transition of $\mathrm{Mn}^{2+}[6]$.

This paper describes a new type of phase transition in this family, marcasite to $\mathrm{CuAl}_{2}$-type, which has been found in $\mathrm{CrSb}_{2}$. High-pressure form of $\mathrm{CrSb}_{2}$ was metastably obtained by quenching from $7-7.7 \mathrm{GPa}$ and $600-700^{\circ} \mathrm{C}$. The structural analysis and electrical and magnetic measurements reveals that the transition accompanies a change of d-electron character from localized to itinerant.

\section{Experimental}

Stoichiometric mixture of chromium and antimony powders was heated at $700^{\circ} \mathrm{C}$ for $168 \mathrm{~h}$ in an evacuated silica tube and quenched to room temperature. The marcasite-type (low-pressure form) sample thus obtained was ground, pressed into pellet, and subjected to high-pressure/ temperature condition using the belttype high-pressure equipment. The detailed cell assemblage of the belt-type equipment is shown in Fig.1. High-pressure/ temperature treatments were carried out at 3-7.7 GPa and 400$700^{\circ} \mathrm{C}$ for $30 \mathrm{~min}$ and then the sample was quenched to room temperature prior to releasing the applied pressure. Phase identification of the recovered sample was performed by powder $\mathrm{X}$-ray diffraction analysis. The crystal structure of high-pressure form was refined by Rietveld analysis of the powder X-ray

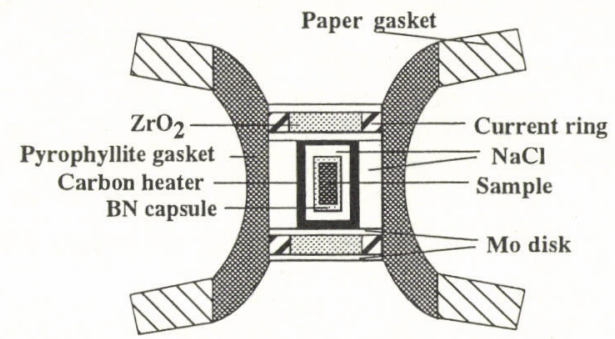

Fig.1. The cell assemblage of the belt-type high-pressure equipment.

diffraction data using the RIETAN program [8]. The magnetic measurements were carried out by a magnetic torsion balance in the temperature range $77-300 \mathrm{~K}$.

\section{Results and Discussion}

Figure 2 shows the $\mathrm{X}$-ray diffraction patterns of products quenched from $600^{\circ} \mathrm{C}$ at various pressures. The detailed $\mathrm{X}$-ray

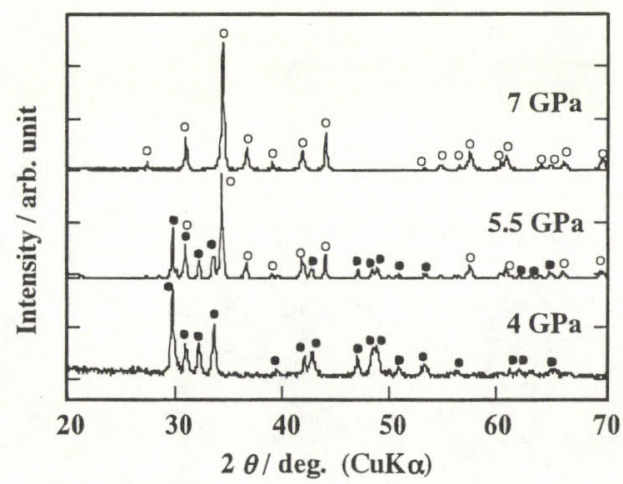

Fig.2. The X-ray powder diffraction patterns of $\mathrm{CrSb}_{2}$ quenched from $600^{\circ} \mathrm{C}$ at various pressures; open circle: high-pressure form, closed circle: low-pressure form. 
diffraction study of quenched samples revealed that the crystal structure of the sample treated below $5 \mathrm{GPa}$ was low pressure marcasite-type, while a new modification of $\mathrm{CrSb}_{2}$ coexisted with the low-pressure form in the samples treated above 5.5 $\mathrm{GPa}$. Single phase of this high-pressure form was obtained after treatments at 7-7.7 $\mathrm{GPa}$ and $600-700^{\circ} \mathrm{C}$.

The high-pressure form is metastable at ambient condition and transforms to low-pressure marcasite-type structure by heating above $300^{\circ} \mathrm{C}$ at atmospheric pressure.

The X-ray diffraction data of the high-pressure form was completely indexed on a tetragonal unit cell with lattice parameters of $\mathrm{a}=6.49 \AA$ and $\mathrm{c}=5.74 \AA$. From the Rietveld analysis, the crystal structure was refined to be body-centered tetragonal $\mathrm{CuAl}_{2}$-type with the lattice parameters $\mathrm{a}=6.4913(3) \AA$ and $c=5.7440(4) \AA$. The final refinement gave the R-factors of $\mathrm{R}_{\mathrm{I}}=5.37 \%$ and $\mathrm{R}_{\mathrm{F}}=4.01 \%$

The crystallographic parameters of the high-pressure form $\left(\mathrm{CuAl}_{2}\right.$-type) are given in Table 1 . The crystal structure is shown in Fig. 3.

The high-pressure form of $\mathrm{CrSb}_{2}$ is isostructural with $\mathrm{TiSb}_{2}$ and $\mathrm{VSb}_{2}$ [9]. At ambient pressure, only two compounds, $\mathrm{TiSb}_{2}$ and $\mathrm{VSb}_{2}$, are known to crystallize into $\mathrm{CuAl}_{2}$-type structure in transition metal diantimonides family. The occurrence of the $\mathrm{CuAl}_{2}$-type in $\mathrm{TiSb}_{2}$ and $\mathrm{VSb}_{2}$ is considered to be due to the relatively large size of the transition metal atom as compared with the other group transition elements [9].

The interatomic distances in high-pressure $\mathrm{CrSb}_{2}$ are listed in Table 2. The local environments of $\mathrm{Cr}$ atoms in bothlow-pressure (marcasite-type) and high-pressure $\left(\mathrm{CuAl}_{2}\right.$-type) structures are shown in Fig.4.

In contrast to the octahedral coordination of $\mathrm{Cr}$ atoms in the low-pressure form, each $\mathrm{Cr}$ atom is surrounded by $8 \mathrm{Sb}$ atoms at equidistance and additionally $2 \mathrm{Cr}$ atoms ( 10 -fold coordination)

Table 1. Crystallographic parameters of $\mathrm{CrSb}_{2}$ (high-pressure form).

\begin{tabular}{ccccccc}
\hline \multicolumn{6}{l}{ Space group: $14 / \mathrm{mcm}$} & \multicolumn{4}{c}{$(\mathrm{No} .140)$} & $\mathrm{a}=6.4913(3) \AA, \mathrm{c}=5.7440(4) \AA$ \\
\hline Atom & Site & Occ. & $x$ & $y$ & $z$ & $B\left(\AA^{2}\right)$ \\
\hline $\mathrm{Cr}$ & $4 a$ & 1.0 & 0.0 & 0.0 & 0.25 & $0.7(3)$ \\
$\mathrm{Sb}$ & $8 h$ & 1.0 & $0.1587(5)$ & 0.6587 & 0.0 & $0.2(1)$
\end{tabular}

final R-factors: $\mathrm{R}_{._{1}}=5.37 \%, \mathrm{R}_{\mathrm{F}}=4.01 \%, \mathrm{~S}=1.3773$

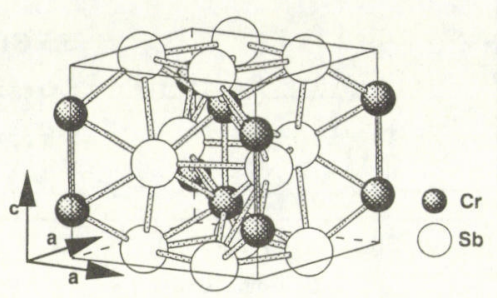

Fig.3. The crystal structure of $\mathrm{CrSb}_{2}$ (high-pressure form).
Table 2. Interatomic Distances in $\mathrm{CrSb}_{2}$ (high-pressure form).

\begin{tabular}{lll}
\hline $\mathrm{C}-\mathrm{Cr}$ & $2.8720(2) \AA$ & $\times 2$ \\
$\mathrm{Cr}-\mathrm{Sb}$ & $2.8339(14)$ & $\times 8$ \\
$\mathrm{Sb}-\mathrm{Sb}$ & $2.914(9)$ & $\times 1$ \\
& $3.325(5)$ & $\times 2$ \\
& $3.455(2)$ & $\times 4$ \\
& $3.535(4)$ & $\times 4$ \\
\hline
\end{tabular}

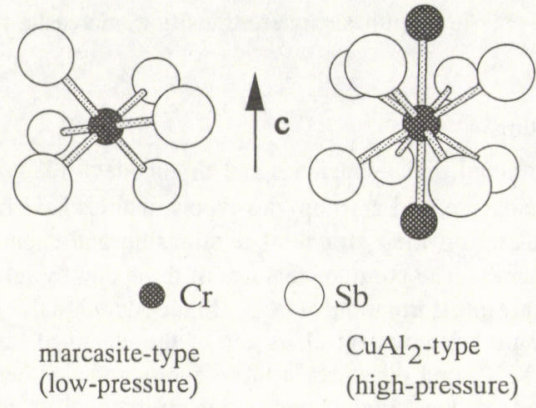

Fig. 4. The local environments of chromium atoms in $\mathrm{CrSb}_{2}$.

in the high-pressure form. As seen in Fig.4, Cr-Cr-Cr linear chains are formed along the $\mathrm{c}$-axis. The $\mathrm{Cr}-\mathrm{Sb}$ distance in the high-pressure form is $2.834 \AA$. This value is longer than that of the low-pressure form and nearly equal to sum of the metallic radii. According to the literature [1], the average $\mathrm{Cr}-\mathrm{Sb}$ distance in the low-pressure marcasite-type, in which the bond nature is believed to be mixed ionic and covalent character, is $2.708 \AA$. The bonding scheme in the marcasite-type phase is explained as $\mathrm{Cr}^{4+}\left(\mathrm{Sb}_{2}\right)^{4}$. The higher coordination number of $\mathrm{Cr}$ atom, the longer $\mathrm{Cr}-\mathrm{Sb}$ bond distance, and the formation of $\mathrm{Cr}-\mathrm{Cr}$ bond indicate the metallic bond character in the high-pressure form.

There is still a possibility of the formation of $\mathrm{Sb}-\mathrm{Sb}$ dimer in the high-pressure form. It is somewhat difficult to estimate the nature of $\mathrm{Sb}-\mathrm{Sb}$ bond. The nearest $\mathrm{Sb}-\mathrm{Sb}$ distance in $\mathrm{CrSb}_{2}$ (high-pressure form) is $2.914 \AA$, while the value is $2.84 \AA$ [1] in the low-pressure form. In fact, $\mathrm{Sb}$-Sb dimeric bonds are believed to exist in isostructural $\mathrm{TiSb}_{2}$ and $\mathrm{VSb}_{2}$ [9]. The $\mathrm{Sb}-\mathrm{Sb}$ bond distances in $\mathrm{TiSb}_{2}$ and $\mathrm{VSb}_{2}$ are $2.840 \AA$ and $2.892 \AA$, respectively [9]. These values are comparable with those of other $\mathrm{TSb}_{2}$ family $(2.82-2.88 \AA)$ with the pyrite, marcasite, and arsenopyritetype structures [1]. However, it should be noticed that the $\mathrm{Sb}$-Sb distance in $\mathrm{CrSb}_{2}$ (high-pressure form) is the longest value among the transition metal diantimonides family $[1,9]$. This fact suggests that the covalent character of $\mathrm{Sb}-\mathrm{Sb}$ bond in the low-pressure form is weakened through the phase transition and the metallic bond character appears in the high-pressure form.

From the structural consideration discussed above, it is concluded that the phase transition of $\mathrm{CrSb}_{2}$, low-pressure marcasite-type to high-pressure $\mathrm{CuAl}_{2}$-type, accompanies a changein bond nature from the mixed ionic and covalent character to the metallic character. The transition accompanies $11 \%$ volume 


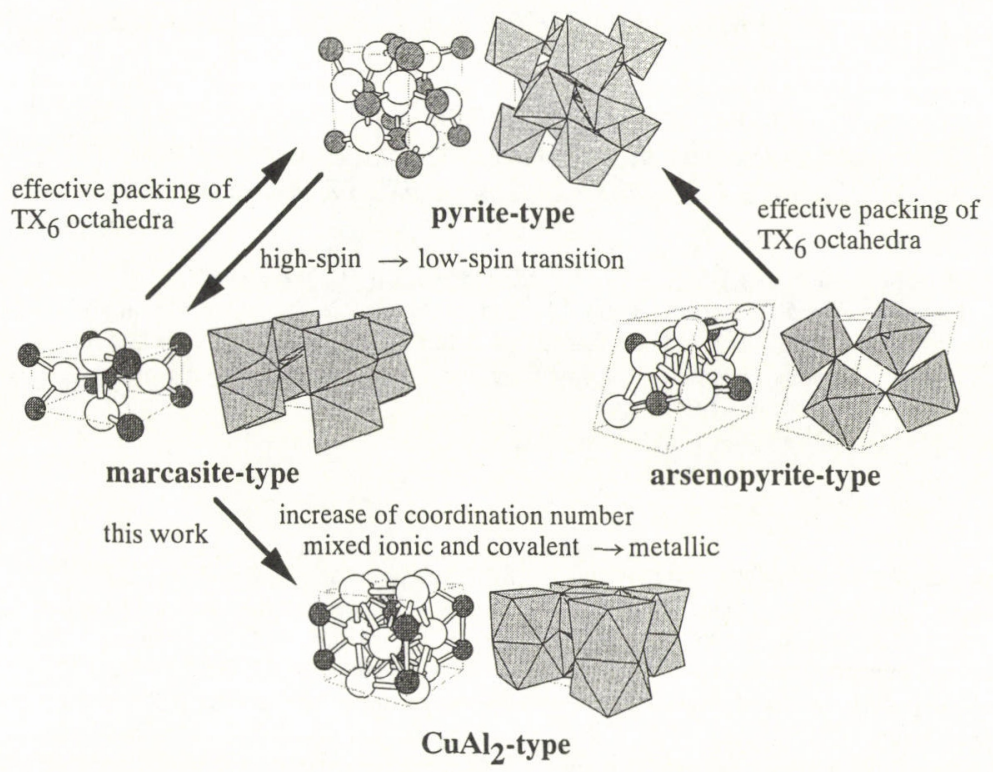

Fig. 5. High-pressure phase transitions of transition metal dichalcogenides and dipnictides, $\mathrm{TX}_{2}$, with the pyrite, marcasite, and arsenopyrite-type family.

decrease.

The low-pressure form of $\mathrm{CrSb}_{2}$ is an antiferromagnetic semiconductor with the Néel temperature of $275 \mathrm{~K}[2,10]$. A high-spin $3 \mathrm{~d}^{2}$ electron configuration for $\mathrm{Cr}$ was proposed. In contrast, The electrical and the magnetic measurements revealed that the high-pressure form exhibited metallic conductivity and itinerant electron ferromagnetic behavior with the Curie temperature of $160 \mathrm{~K}$, ie., the localized character of the lowpressure form changed to itinerant in the high-pressure form.

Figure 5 summarizes the high-pressure phase transitions in transition metal dichalcogenides and dipnictides with the pyrite, marcasite, and arsenopyrite structures. The maracasite-type to $\mathrm{CuAl}_{2}$-type transition, found in $\mathrm{CrSb}_{2}$, represents a new transition scheme in this family, that is, nonmetal-metal transition associated with the change in bond nature. This suggests possible synthesis of new metallic compounds in this family by applying highpressure technique.

\section{Acknowledgments}

This work has been supported in part by a Grant-in-Aid for Scientific Research (B)(2) (No. 09450238) from the Ministry of Education, Science, Sports, and Culture, Japan.

\section{References}

[1] G. Brostigen and A. Kjekshus, Acta Chem. Scand., 24, 2993 (1970).

[2] H. Holseth and A. Kjekshus, Acta Chem. Scand., 24, 3309 (1970).

[3] T. A. Bither, C. T. Prewitt, J. L. Gilson, P. E. Bierstedt, R. B. Flippen, and H. S. Young, Solid State Communications, 4, 533 (1966).

[4] P. C. Donohue, T. A. Bither, and H. S. Young, Inorg. Chem., 7, 998 (1968).

[5] H. Fjellvåg, A. Kjekshus, T. Chattopadhyay, H. D. Hochheimer, W. Hönle, and H. G. Von Schnering, Phys. Lett., 112A, 411 (1985).

[6] T. Chattopadhyay and H. G. Von Schnering, J. Phys. Chem. Solids, 46, 113 (1985).

[7] H. Fjellvåg, W. A. Grosshans, W. Hönle, and A. Kjekshus, J. Magn. Magn. Mater., 145, 118 (1995).

[8] F. Izumi, in "The Rietveld Method" (R. A. Young, Ed.), chap.13, Oxford University Press, Oxford, 1993.

[9] J.D. Donaldson, A. Kjekshus, D. G. Nicholson and T. Rakke, J. Less-Common Metals, 41, 255 (1975).

[10] A. Kjekshus, P. G. Peterzens, T. Rakke, and A. F. Andersen, Acta Chem. Scand., A33, 469 (1979). 